\title{
REVISIÓN/REVIEW
}

\section{DE PERFILES Y PERSONAJES: EVOLUCIÓN DE LA REPRESENTACIÓN DEL USUARIO EN LAS REDES SOCIALES Y LOS JUEGOS DE ROL}

Manuel Hernández Pérez¹: Universidad de Murcia. España.

mhp2@um.es

\section{RESUMEN}

Los juegos de rol en línea constituyen, junto a las redes sociales, las opciones más populares de ocio en la red. En el presente estudio, se sitúa el origen de estas plataformas en la evolución digital de las narrativas colaborativas y juegos de rol. Para ello, se examina la evolución del Juego de Rol en sus diferentes adaptaciones digitales a la vez que se presenta la evolución de otras formas narrativas a través de la introducción del hipertexto. Finalmente se analiza la imagen proyectada por el usuario en estas narrativas, en las que el perfil de una red social es tratado "como un personaje más".

PALABRAS CLAVE: Juegos de rol - Narrativa - Hipertexto - MMORPGs - Redes Sociales

\footnotetext{
${ }^{1}$ Manuel Hernández Pérez: Universidad de Murcia. España. 


\title{
PROFILES AND CHARACTERS: \\ EVOLUTION OF THE REPRESENTATION OF THE USER IN SOCIAL NETWORKS AND ROLE-PLAYING GAMES
}

\begin{abstract}
Role-playing games -along with social networks- have become the most popular leisure activity online. This paper locates the origin of these web platforms in the digital evolution of collaborative narratives and role-playing games. In order to do so, it studies the evolution of the role-playing game through its different digital adaptations and presents the evolution of other narrative forms through the introduction of hypertext. To end up, it analyzes the image projected by the user in these narratives, where a social network profile is considered as a character itself.
\end{abstract}

KEY WORDS: Role Playing Games - Narrative - Hypertext - MMORPGs - Social Networks

\section{LOS ESPEJOS DEL "YO" EN EL OCIO ACTUAL}

La evolución de la conciencia del "yo" es indisociable de la propia historia de la modernidad. En palabras del historiador americano Warren Susman (1984, p. 271), "modernidad" no es otra cosa que "el desarrollo de la conciencia del yo". Si para este autor, el desarrollo del cómic, la radio y el cine no es comprensible sin un desarrollo del "yo", en términos de "definición, problemática y contexto para su desarrollo", para nosotros es aún más evidente el papel que juega este concepto en el breve pero intenso periodo de evolución de Internet: desde el inmenso catalogo digital a la red 2.0, reflejo de lo social y personal.

Más de veinte años después de los primeros estudios de Turkle $(1984 ; 1995)$ sobre los usuarios de las redes de juego, comenzamos a tener una perspectiva más que suficiente acerca de la necesidad que tiene el hombre de definirse en ámbitos tan diferentes como el trabajo o el ocio. Las personas se enfrentan día a día al reto, más difícil de lo que parece, de identificarse y definirse, deseosos de aportar algo a esa "pantalla global" que define Lipovetsky (2009). El mundo del ocio en la red no escapa de esa tendencia, presentando cada vez historias más personalizadas, en las que el usuario tiene mucho que decir. Los personajes, los objetivos e incluso el espacio y el tiempo de la narración, ya no son elementos estáticos sino adaptables. Esta configuración de los componentes del relato no es nueva, puesto que encontramos precedentes en las narrativas colaborativas (NC), así como en el género lúdico. En este terreno se señala la aparición del juego de Rol (JdR) como punto de inflexión pues presenta características comunes a la narrativa y al género lúdico.

El primer juego de rol, creado por Gary Gigax en 1974, ya dió la oportunidad a los jugadores de construir una historia y unos personajes e incluso influir en el ritmo de la narración. Recogiendo componentes del juego clásico como la Aleatoriedad o el 
Mimetismo (Caillois, 1958), se construyeron sistemas reglados, para la narración de un relato entre usuarios. El videojuego, casi coetáneo al juego de rol, ha ido agregando a la experiencia de juego dimensiones sociales, representacionales y lúdicas, características que definen al Videojuego de Rol (VJdR) y al MMORPG (Carr et. col, 2006) así como al tradicional juego de rol de papel y lápiz (Dormans, 2006).

Otro aspecto común a ambas plataformas, definitorio del juego de rol (JdR) y el videojuego de rol (VJdR) es la capacidad que otorga al usuario de experimentar la creación de mundos. No es gratuito que Aarseth $(2007$, p.4) los incluya ambos como "juegos en entornos virtuales", dejando sin embargo fuera a otros productos digitales más cercanos a la mera simulación como son los videojuegos de cartas.

El presente texto describirá la ruta que podrían haber seguido estas Narrativas de colaboración que han transcendido al género lúdico y enriqueciéndolo, han formado nuevos géneros híbridos, en los cuales el concepto de "yo" en todas sus acepciones juega un papel central. Nos referimos obviamente a los "perfiles" de las redes sociales personales (nuestra presencia en MySpace, Hi5, Facebook, YouTube, etc.) en las que se recogen datos de perfiles sociodemográficos, psicográficos y un mapa de los nodos de nuestra red social.

Como veremos, los juegos de rol han supuesto un precedente de otros productos digitales de ocio tan diferentes como World of Warcraft (2003) o Facebook (2004). Sin embargo ambas tipos de plataforma, como redes sociales virtuales, presentan importantes similitudes y un mismo objetivo: la creación de experiencias a través de lazos sociales y la narración colaborativa. Así se hace tangible que la propia definición del "yo" pasa por la construcción de una personalidad social que es vista como un objeto en una red social (una página personal o perfil). Estas conexiones, ya existentes en una era pre-digital, definen al hombre como un ser social, arbitrando las relaciones entre el altruismo y el castigo, los deseos y las repulsiones, lo que en definitiva equivale a renombrar al ser humano como homo dictyous u "hombre en red" (Christakys y Fowler, 2010).

\section{VINCULACIÓN DE LA NARRATIVA CON EL JDR TRADICIONAL}

\subsection{El Juego como Relato: Características del JdR}

El juego de rol no aparece oficialmente hasta 1974 con la popular obra de Gary Gygax y Dave Arneson, "Dragones y Mazmorras". Sin embargo algunos de sus componentes, como son la imitación gestual, la representación con maquetas, planos o figuras, la asunción de roles o papeles, eran ya parte de los juegos. La idea de cambiar la historia, por ejemplo, cuando el argumento de un juego se encuentra enmarcado en un contexto histórico podría haber sido obtenida de wargames tales como Gettysbourg, comercializados desde 1954 en EEUU.

Nosotros entendemos por juego de rol, todo aquel en el que las posibilidades de proyección e identificación con el personaje interpretado se maximizan en un marco lúdico reglado. Nótese que por ello no deja de perderse el componente de azar que 
caracteriza a toda actividad lúdica. El jugador posee un máximo control sobre lo que su personaje es, pero no sobre las circunstancias en las que se desenvuelve. A este respecto, la figura del Director de Juego (DJ) es una especie de dios o guionista supremo, pues dicta a los personajes sus opciones en el mundo creado/presentado y las consecuencias de sus actos. En esta retroalimentación constante, en este continuo fluir de la información, se moldea el personaje y es, por tanto, no sólo el DJ sino también el jugador, quien explota al máximo sus recursos creativos. Son ambos quienes dan forma conjuntamente a este relato interactivo.

Las circunstancias que rodean al juego de rol, desde su misma creación como divertimento (un grupo de personas frente a un narrador, interrogando, inquiriendo y dando forma a la historia), a la complejidad de los universos narrativos o mundos textuales en que se basan (normalmente inspirados novelas y películas de género fantástico), sitúan a éste como un interesante ejemplo híbrido de literatura y juego. Siguiendo a R. Caillois y su célebre Théorie des jeux (1958), intentaríamos situar al juego de rol, como consecuencia de la evolución de la Paidea que traduciremos por "fantasía de divertimento" en sus diferentes formas regladas. Esta tendencia al orden, o manual de todo juego supone otro polo que define el juego (Ludus). El autor define entonces diferentes tipos de juego tales el Agôn, el Alea, el Mimicry, y el Ilinix. El Juego de Rol, como veremos, no es fácilmente asimilable a esta tipología, por lo que bien podría entenderse como una descripción de los componentes que pueden encontrarse:

AGÔN: éste se nos define como Competición entre los jugadores, que pese a mostrar inicialmente basada en la igualdad de oportunidades, persigue demostrar la superioridad de las cualidades del vencedor, su logro dará la gloria y será signo de reconocimiento de su excelencia en el terreno. Dicha "búsqueda artificial de la igualdad de oportunidades", como nos señala el autor "manifiesta el principio esencial de la rivalidad". Sin embargo el JdR es sobre todo relato, y el objetivo último de éste no puede ser múltiple, como sugiere el Agôn (ya que da tantos finales diferentes a la historia como competidores pueden lograr el triunfo). El relato del JdR es relato cooperativo y ni siquiera el fracaso de los jugadores supone el triunfo del Director de Juego (DJ), pues como narrador, es ajeno a los beneficios de la victoria. Teóricamente, el DJ, sólo puede estar de parte de los jugadores porque de estar en su contra, podría construir una aventura insuperable, un relato que no se desarrollaría "correctamente". En términos de la semiótica estructural de Entrevernes (Grupo Entrevernes, 1982), ambos grupos, DJ y jugadores, tienen como objetivo común la consecución de un Programa Narrativo (PN), o lo que es lo mismo, una sucesión y encadenamiento de estados y transformaciones de entre los posibles.

ALEA: Se define como aspecto contrario del Agôn, y pasa de ser un juego que viene de la decisión y las capacidades del jugador, para dar al azar el papel decisor. En el JdR es por medio del dado como se decide el éxito o fracaso de la acción, así como el curso de otras aleatoriedades, de entre las previstas por el manual de juego. De tal forma que aunque siempre existe un margen no recogido por las reglas (en el que es el DJ quien tiene la última palabra), normalmente las tiradas de dados deciden en términos totales o parciales. 
Hasta aquí se han descrito las fantasías ligadas al control del mundo. Aquellas en las que el Ludus posee mayor influencia sobre la Paidea. Pero existen otras formas en las que la fantasía consigue imponerse con mayor fuerza:

En la MIMICRY, palabra inglesa que designa el mimetismo, la capacidad del sujeto para fundirse con el ambiente, el sujeto prefiere crear un personaje que no gobierne el mundo ni se enfrente a él, sino que le sirve para integrarse en ese universo fantástico. La victoria es entonces la muerte aparente de su "ser real", al que sumerge en letargo mientras asume la nueva identidad. Mimicry guarda relación con la mimesis griega, a través de la cual nos deleitamos en la recreación de los logros del mundo natural buscamos filiaciones y las capturamos, haciéndolas nuestras. Callois señala que la Mimicry se basta para presentar todas las características del juego tal y como todos lo entendemos: la libertad, convención, suspensión de lo real, espacio y tiempo delimitado. El JdR se basa, como su nombre indica, en la asunción de identidades o roles, las de un mundo fantástico residente en la narración conjunta del Director de Juego (DJ) y los Personajes Jugadores (PJs). Es por ello que un buen DJ considera su obligación el velar por la interpretación de las identidades o papeles. Como árbitro teatral de esta interpretación, asume que no sólo acota las acciones posibles basándose en las capacidades de los personajes, (que por otra parte son objetivas y obedecen a un sistema reglado por el manual o ludus). Efectivamente, su deber es también restringir la libertad de los jugadores, reglando el comportamiento y las decisiones de sus personajes, que deben ajustarse a un código moral, resumido en lo que ese personaje haría en esta situación.

Finalmente se señala que el juego es adicción, es deseo. Ese componente emocional puede provocar la suspensión de la realidad y la emergencia de una "conciencia lúdica". Este estado que se define como "vértigo o pánico voluptuoso" (Caillois; 1958, p.61) el que nos viene dado por el Ilinix, un "torbellino de agua" que limita todo objetivo al juego mismo, como único marco.

\subsection{Definiendo la Narrativa Liquida}

Definir las formas actuales de narrativa, una transformación acometida a lo largo de la Historia, plantea un problema de complejidad similar al de la descripción de la sociedad en que vivimos. Ambas, la narrativa y la sociedad, vienen marcadas por una constante búsqueda de la individualidad fundamentada en la diferencia. Esa búsqueda, además, lleva a un planteamiento permanente de la identidad fragmentada y global, por lo que el mejor término para definir la cultura en está sociedad es precisamente el de "hibridación" (Bauman 2006). Este concepto puede guiar desde una perspectiva general el camino recorrido por la narrativa desde el siglo XIX. Este recorrido, como señala Ryan (2004), viene señalado por la dualidad marcada entre el efecto de inmersión (la recreación de un mundo) y el de interacción (el juego que ejecuta el lector), si bien podríamos decir que tras el cambio que supone la hibridación, han aparecido nuevas formas narrativas con la capacidad de asimilar esos dos efectos de manera simultánea.

La segunda pieza clave de este proceso viene dada por la introducción de la 
interactividad, aspecto indisociable de la narrativa desde la aparición del hipertexto. El hipertexto es la expresión de una nueva dimensión en la comunicación, cuya geometría se conforma con un entramado de hilos textuales o "textos plegados" tal y como los define Rodríguez de las Heras (2006). El hipertexto se beneficia de la tecnología para recorrerlos de una forma inmediata, si bien su soporte no es necesariamente digital dándose casos de literatura hipertextual anteriores a la aparición de estos soportes (Koskimaa, 2000; Vega, 2003). Como consecuencia de la consolidación del hipertexto se define un nuevo lector. Este nuevo lector puede dirigir el texto, o más bien, el sentido de su lectura. A esta caracteristica de lectura interactiva puede sumarse el carácter "ergódico" o moldeable de un texto (Landow, 1993; Aarseth, 1997) en cuyo caso permite al usuario transformarlo.

A estos rasgos colaborativos del texto se une un fenómeno basado en la reticularidad del mismo. Esta "red de información" permite al lector recorrer los nodos del hipertexto creando una experiencia literaria personal que entendemos como narrativa emergente. La incorporación de otros lenguajes (principalmente gráfico y audiovisual), lo que transforma el hipertexto en hipermedia, un "maridaje íntimo, integrado por el hipertexto y los multimedia" (Mabrouk et al, 1991).

En definitiva, podríamos decir de esta literatura digital o "Narrativa Liquida", emulando la terminología de Bauman, que vendría definida por la reticularidad, la aleatoriedad (adaptación del concepto de juego) y la inmersión. Si bien el efecto de la aparición de esta Narrativa Liquida, lejos de una simple evolución marcada por la tecnología, viene a señalar un cambio importante en la manera de concebir la sociedad y los procesos de socialización. En ese sentido, la literatura digital no es sólo una masterización de habilidades mecánicas, sino un nuevo sistema que nos permite usar la tecnología con propósitos de acción social y mantenimiento de la identidad (Martin, 2009).

\section{EVOLUCIÓN DEL GÉNERO LÚDICO: UN CORTO CAMINO ENTRE EL JDR Y EL VJDR}

En cada revolución tecnológica la narrativa es transformada como consecuencia de la incorporación de nuevos soportes que modulan la relación entre nuestros sentidos y la información procesada. Durante un largo período de tiempo, por ejemplo, el conocimiento ha impuesto una configuración lineal y secuencial del texto, determinada por la propia tecnología del soporte-libro que guía el sentido de la lectura y centraliza el proceso de conocimiento en torno a un único medio (Martín-Barbero, 2003).

Esta idea, podría ser trasladada al terreno del juego en el que nos encontramos con importantes cambios con la introducción de soportes digitales o videojuegos. En estos, por basados en los hipermedia (Klevjer, 2007), descansa la idea subyacente del uso recurrente de otros medios y lenguajes (audiovisual, ilustración, texto y cómic...), así como de la construcción de una narrativa no lineal y dirigida por el usuario. En esa aproximación basada en el medio se encuentran los teóricos que ven el VJ como una evolución de los lenguajes audiovisuales, especialmente el cine (Manovich, 2001). Así, el videojuego heredaría del cine la utilización de los montajes temporal y espacial, la realización multi-cámara y la utilización de los efectos en un lenguaje cargado de 
sentido. Para este último aspecto es fácil encontrar ejemplos en los shooters como Gears of war (Epic Games, 2006) donde el uso del blanco y negro en la película "dramatiza" los últimos segundos de vida del personaje. Además, el videojuego se comporta como un género híbrido y acude a la utilización de lenguaje audiovisual en películas intersticiales, en las que se suspende la capacidad de interacción del usuario, para presentarnos una narración secundaria o introducirnos mejor en la historia.

\subsection{Transformaciones del Juego de Rol (JdR) en el entorno digital}

Videojuego de Rol (VJdR) es el término por el que designamos a los videojuegos basados en juegos de rol o que han sido concebidos para adaptar su lenguaje y/o el universo narrativo que lo inspira. En términos generales, ambos subgéneros podrían definirse como actividades lúdicas basadas en la construcción de un juego del que emerge un relato, así como en la creación de personajes configurables. Si bien se señala que no existen pocas variedades en ambos subgéneros lo que hace complicado la descripción de una tipología. En el JdR tradicional, por ejemplo, existen diferencias significativas desde el JdR de mesa, los basados en cartas y otras variedades de JdR interpretativo. Por su parte los VJdR también poseen una desarrollada tipología que podrían además incluir otras formas de videojuego con características de JdR o VJdR. En el camino entre ambas plataformas (el JdR y el VJdR), son muchas las transformaciones que tienen lugar, tanto en el relato como en la experiencia de juego (gameplay):

- Existe un guión, un macro-texto, diseñado expresamente para ser jugado. En ese sentido, ambos subgéneros, JdR y el VJdR, comparten esquemas narrativos recurrentes, normalmente inspirados en la literatura de fantasía épica, ciencia ficción y otros géneros de literatura fantástica. En ellos distinguimos claramente personajes y objetos, así como un argumento, donde objetos y personajes se someten a relaciones de causalidad. Estos son junto con la caracterización de personajes y la creación de mundos, los principales elementos del juego de rol así como de otros juegos con tintes narrativos (Tosca, 2000; Jørgensen, 2010).

- El autor del texto en el JdR es el Director de Juego (DJ), quién ha programado la partida y dirige el juego. Éste ejecuta las tareas de Emisor/Locutor del texto que constantemente se "reescribe". En cambio en el videojuego, esta figura es suplantada por diseñadores y programadores que han previsto las posibilidades de la historia de una forma limitada y dan verdadero sentido al término "programación". Este ejercicio establece una relación inversa entre interactividad y narratividad que conocemos como "dilema interactivo" (Berenguer, 2007).

- El texto de los videojuegos de rol está enriquecido con otros lenguajes. Pero el Juego de Rol ya se apoya en otros textos (conversaciones, introducciones...), gráficos (mapas, escenarios,...) y diferentes medios de naturaleza audiovisual (cut-scenes). El esquema de comunicación ahora implica una comunicación no directa, sino mediada, entre los agentes de comunicación (Contreras, 1998).

- El componente azaroso, el alea propio de todo juego, cobra en el marco del juego 
de rol un papel especial de mediador. Así, muchas de las decisiones del juego, que no dependen directamente del DJ, son resueltas por la puntuación obtenida en una tirada de dados. En el entorno digital, el papel regulador es ejercido por la inteligencia artificial y el marco reglado es el propio programa informático.

- La duración de la partida del VJdR es, emulando al JdR, cada vez mayor ya que por lo general se trata de juegos con una gran vida lúdica. Por ejemplo, la última entrega de la popular saga de Square Enix, Final Fantasy XIII (PS3, 2010) requiere entre 50 y 60 horas para ser jugado. En ambos casos, entendemos la partida en sentido discursivo, ya que el texto (la partida jugada hoy, la llamada "sesión") es sólo una parte del discurso (la "aventura", o narración que supone toda la partida) que será jugada en varios días.

- El personaje o personajes, son absolutamente configurables desde el inicio de la partida. El jugador participa así en el relato construyendo al personaje según sus preferencias, aunque el resultado que observamos en su aspecto físico, sus habilidades e incluso su personalidad, deberán ser adaptados conforme a unas normas, generalmente basadas en sistemas de puntuación, tablas y el mismo universo mitológico recreado en el juego. Estas leyes quedan recogidas en los manuales de jugador y DJ, llegando a ser en ocasiones de cierta complejidad, por lo que sólo son estudiadas por aquellos jugadores que desean desempeñar funciones de Director de Juego (DJ). Este proceso de customización de los personajes es continuo, se actualiza entre sesiones o partidas completas, por acuerdo entre los jugadores y el DJ. La adquisición de equipamiento, sin embargo puede darse en cualquier momento, reglada siempre por las normas del JdR. En el videojuego de rol, la actualización es normalmente automática y es, en ocasiones un factor clave para alcanzar un estado (vencer a un fuerte enemigo, adquirir un conocimiento o técnica "mágica"...), e influye decisivamente en el avance de la narración.

Para concluir se señala que el proceso de comunicación resultante de la partida de rol tradicional produce un nivel de representación que exige mayor implicación por parte del jugador. La información visual/verbal del Director de Juego construye una suerte de "efecto de inmersión indirecto". En el videojuego, en cambio, esta información es directa e inmersiva, predominantemente visual.

\subsection{Aspectos Narrativos en el VJdR}

El debate sobre los aspectos narrativos del videojuego se inicia con la reflexión de Eskelinen (2001). Este autor, apoyado en la tipología del cibertexto de Aarseth (1997) señala que el videojuego es una práctica configurativa y no interpretativa (al contrario que la literatura y el cine). Por tanto, para él es lógico que los componentes narrativos no sean otra cosa que "meros adornos" que restan valor a otras experiencias asociadas al juego (Eskelinen, 2001). A ese respecto se observa que el texto del videojuego es principalmente ergódico y que es este valor lo que capacita al lector a moldear su materialidad y lo convierte en un co-escritor del mismo, abandonando su papel habitual de lector (Aarseth, 1997). Estas posiciones cercanas a la lúdica que en ocasiones se conocen como radicales, se caracterizan por una fuerte crítica al uso de 
las herramientas de la Teoría Literaria y la Narratología. Para estos autores (Juul, 2001; Eskelinen, 2001; Frasca, 2003) el juego y más concretamente el videojuego, debe ser estudiado desde una perspectiva propia, libre de la influencia de otras disciplinas, y siempre desde un marco lúdico.

Nuestra postura no niega el importante peso que la narrativa tiene en el juego de rol y en el videojuego, especialmente en ciertos subgéneros, si bien los sigue considerando como géneros lúdicos con fuertes componentes narrativos. Por otra parte, la importancia que estos relatos tienen en el desarrollo del juego es un aspecto bien estudiado entre los teóricos así como su principal argumento para la utilización de metodologías propias de la narrativa en el análisis de estas formas de género híbrido. Así, distinguiríamos tres formas en las que un relato puede tener presencia en un juego de rol, tanto en su versión tradicional (JdR) como digital (VJdR):

\subsubsection{Narración Anidada}

En el desarrollo de una partida de Rol la interacción del usuario no es siempre necesaria. Los directores de Juego (DJ) actúan no sólo como arbitros del juego, sino como narradores. Ellos dan comienzo al relato colaborativo que es la partida y no dudan en apoyar su narración en diferentes medios o soportes: mapas, planos, figuras que representan personajes (principales o antagonistas), música, efectos de sonido, máscaras u otros elementos de atrezzo, etc. Así, en ciertos momentos, el juego debe ser suspendido para que la narración avance.

Estos episodios sin interacción también se dan con frecuencia en el ámbito del videojuego mediante los denominados intersticios o cut-scenes. Éstos, normalmente siguen códigos cinemáticos en forma de películas de imagen real o animación, aunque pueden ser también basados en otros lenguajes secuenciales parecidos al cómic como en el caso de Max Payne (2001) o incluso dotados de cierta interactividad limitada (Assasin Creed, 2007). El propósito original de esta técnica es reforzar el componente narrativo del juego dotando de coherencia a la historia narrada a través de las pantallas (stages). Además las cut-scenes ubican al lector en una situación relacionada con las consecuencias de sus acciones, o en el contexto en que se desarrollará su comportamiento.

Este compendio de técnicas suele ser criticado por disminuir la experiencia de juego (gameplay), sobre todo por aquellos autores pertenecientes a una corriente lúdica radical (los ya citados: Eskelinen, 2001; Juul, 2001 o Frasca, 2003) que definen el juego como una experiencia ergódica que no debe ser limitada por la utilización de un discurso narrativo. En el otro extremo, también existen autores que señalan cómo mediante las cut-scenes el esfuerzo ergódico del usuario adquiere un verdadero significado, añadiendo información sobre el mundo ficcional que representa y modulando el ritmo de la experiencia de juego (Klevjer, 2002).

\subsubsection{La Historia de Fondo. La narración como telón de fondo}

Los juegos de rol se inspiran en universos ficcionales propios de la literatura 
(usualmente fantástica), el cine o el cómic. En el caso del videojuego y debido a su naturaleza audiovisual, suelen existir mayores similitudes con películas de género que con las novelas. La razón podría encontrarse en una destilación de elementos descriptivos del texto literario, que realizados en formato audiovisual, pueden servir a una narración más concreta, de carácter estético. El videojuego ofrece en cambio una experiencia complementaria de inmersión y diálogo con el texto, además del placer de asumir las atractivas identidades de los personajes de una historia de éxito. Por otra parte, ese emparentamiento también puede responder a cuestiones de marketing, pues el producto fílmico es más popular y goza de mayor audiencia. Además, es una constante en la historia del videojuego el desarrollo de productos paralelos a las películas, como productos de una misma marca de ficción. Esta cuestión, que se plantea en la actualidad como una estrategia transmedial, ha sido clave en la difusión de películas blockbuster y videojuegos en los últimos 30 años. Un ejemplo interesante de este fenómeno sería la saga de Star Wars iniciada en 1977 por George Lucas que, además de multitud de videojuegos de diferentes géneros, ha inspirado también juegos de rol (JOC Internacional, 1990-1997) y videojuegos de rol (Star Wars: Knights of the Old Republic, 2003-2008).

\subsubsection{Secuencia de Eventos: La narración emergente}

Las misiones o quest son probablemente la mayor aportación del JdR al videojuego, tanto en términos de diseño como de experiencia de juego. Además, son las principales formas de introducir elementos de storytelling inmersos en la experiencia de juego (Tosca, 2003). Es un hecho observable que principalmente en los juegos basados en historias, entre los que se encuentran los JdR y VJdR, la coherencia narrativa o diégesis no depende sólo del uso de construcciones retóricas sino que "emerge" también de la representación de eventos aislados, mediante un proceso de mimesis. Estos eventos los constituyen un sucesión de misiones o quest que podrían ser definidos como acciones orientadas a fines concretos (Tronstad, 2001). Estos eventos 0 acciones son producto de la interacción entre jugadores y deben distinguirse de los "relatos" en que pueden convertirse cuando los actos son narrados. Esta distinción entre acciones del personaje y eventos "representados" es básica, pues señala cómo pueden coexistir la doble naturaleza del JdR y su evolución digital (VJdR), constituyendo una forma genuina de género lúdico y narrativo. En la historia del VJdR, el paso posterior a la incorporación de las quest fue la creación de verdaderos relatos reticulares los cuales podían estar constituidos por historias que a su vez anidaban otros relatos independientes. En estos videojuegos, las misiones pueden ser jugadas sin un orden establecido, pasando del concepto de stage o pantalla estática al de misiones independientes, lo que supone una migración del concepto de "historia" al de "mundo" (Garín y Pérez, 2006). La historia que el usuario experimenta emerge así del juego, transformando un acto en una narración, y en esta narración los eventos que la constituyen son reordenados según la experiencia de juego de cada usuario. Estas "narrativas infinitas", con limitada línea argumental son propias de juegos que no son necesariamente de rol. Un conocido ejemplo lo encontramos en la saga Grand Thief Auto (Rockstar Games, 1997-2010), que experimenta con diferentes formas de asimilar la estructura de quest a una narración lineal. Sin embargo, existen abundantes ejemplos como en el videojuego GTA 3, 
paradigma del juego emergente, donde la interactividad se somete pese al gran abanico de episodios accesorios, a una "estructura principal invariable" (Peinado y Santorum, 2004, p.7). Este aspecto nos hace pensar que no siempre el concepto de "mundo" no está necesariamente reñido con el de relato o "historia".

\section{REDES DE JUEGO, REDES SOCIALES Y OTRAS COMUNIDADES}

En el origen de las Redes Digitales se planteaba una importante duda acerca de si la tecnología nos conduce a modelos de interacción social sin sociabilidad. Diferentes autores (Breton, 2000; Bauman, 2005) planteaban cómo una sociedad fuertemente conectada por medio de la tecnología se dirigía paradójicamente a una situación de pocos encuentros y diálogos. Actualmente, esa idea ha ido cambiando y no son pocos los autores que señalan la importancia de la interacción social entre usuario en el triunfo de los MMORPG (Manninen, 2002; Tosca, 2002; Tychsen et al. 2006), así como ésta, en combinación con la experiencia positiva, es el principal factor en la lealtad de los consumidores en aquellas redes que requieren suscripción (Choi y Kim, 2004).

En la evolución entre plataformas vemos también como cambia la relación entre los elementos narrativos, sociales y lúdicos. En un primer momento, las plataformas digitales priorizaron la comunicación entre nodos de la red, por encima de la experiencia de juego e incluso de la experiencia narrativa. Los chats y comunidades virtuales actualizaban esas redes que ya existían en las sociedades humanas. Más tarde, la red trasladaba el placer de la suspensión de la realidad (Paidea o "Fantasía de divertimento") a estas plataformas, creando las primeras redes de juego, las MUD s y las comunidades de juegos arcades (MMOG). Ambas plataformas darían lugar tras una evolución tecnológica a los actuales MMORPGs. Pero el usuario necesitaba de la construcción de un relato propio que aunase la definición de la identidad y permitiese realizar funciones de socialización. Así, el mundo-juego pasa a ser sustituido por una representación del mundo real y el avatar y los personajes de su juego son sustituidos por una imagen más acorde con estos propósitos, adoptando la forma de perfil personal.

\subsection{Distinción y semejanzas entre Redes Sociales y MMORPG: del Diseño de Personajes a la Creación de Perfiles}

Toda historia tiene personajes, y éstos son los que llevan a cabo o reciben una acción. Este principio puede ser trasladado al componente narrativo del JdR y sus versiones digitales (VJdR y MMORPG), y más aún a otras formas de relato como las que se llevan a cabo, de forma implícita en las redes sociales del ciberespacio. Al igual que el mundo de la ficción, la proliferación de espacios de comunicación en la red ofrece infinitas posibilidades relacionadas con nuestra formación, nuestras inquietudes y/o necesidades. Éste es un punto clave de la cuestión, ya que, para cualquiera que desee integrarse en este nuevo mundo, debe haber una motivación que le haga abandonar su cuerpo. La asunción de esta nueva entidad/instrumento tendrá como consecuencia, entre otras, la incorporación de una nueva identidad virtual, acorde con los requisitos de este espacio. La identidad virtual es imprescindible para lograr con éxito la comunicación en el ciberespacio, nutrirse de información sólo será posible adoptando 
dicha máscara. Pero este proceso supone una influencia simétrica entre ambas partes, de tal forma que es imposible entender la evolución del sujeto y la asunción de diferentes identidades sin el desarrollo del espacio que las justifica, que a su vez es modificado por las necesidades del sujeto. Así, serán dos las vías que tendrá el sujeto para lograr esa acomodación con el nuevo espacio, proceso que asegura su supervivencia en la red: la elección del nido y la creación de un nuevo cuerpo:

\subsubsection{La elección del nido}

El ciberespacio es la evolución lógica de la semiosfera de Lotman (1996). Como macrosistema, no es global ni integrado y como señala Contreras (1998), no es una simple asimilación tecnológica de la biosfera, sino un macrosistema "fragmentado e interconectado". Sus elementos son millones de sistemas de información, y su organización no es jerárquica sino horizontal. El termino ciberespacio que fue inventado por W. Gibson (1986) en sus relatos, no se refería al actual internet ni a las comunidades humanas que la utilizan. Gibson se refería a un concepto, aún irrealizable, de conexión directa entre la red y el usuario por medio de conexiones simbióticas de neuronas y nodos electrónicos. Este visionario autor parece saltar el proyecto social actual, que algunos bautizaron como Espacio Barloviano (Featherstone y Burrows, 1995) y que se encuentra representado por las comunidades de chats, los MUDs, los MMORPG, y otras formas de organización social.

Llegados a este punto, debemos redefinir los papeles del usuario/jugador que, abandonando el terreno del juego-ficción, ese texto ergódico que le permitía "crear mundos", se adentra en el ciberespacio (mundo virtual) de la nueva red. Esta web 2.0, es principalmente una plataforma de contenidos a los que todo usuario tiene acceso y capacidad de interactuar (read-write web). Las comunidades virtuales que en ella proliferan podemos considerarlas como "comunidades de sentido" (Fernback, 1999), que se articulan como un sistema cognitivo y afectivo complejo que se nutre de la experiencia y los significados compartidos. Esta emigración lleva al sujeto/usuario a dos situaciones no tan diferentes: una, la creación de una nueva situación de comunicación mediada que emula la realidad social (Redes Sociales Virtuales). La otra, no exenta de intercambio social y comunicación, se fundamenta en la suspensión de la realidad (Paidea) por medio de la recreación de un mundo de ficción (MMORPG, o Comunidades de Juego).

Pero, ¿qué factores llevan al usuario a la elección de un nido en particular? Dicho de otro modo: ¿A qué se debe la popularidad de sitios como Facebook o World of Warcraft? Este proceso de migración responde a diferentes factores (Ricaurte y Ortega, 2008) entre los que destacan las características del usuario (perfil socio demográfico), de la plataforma (accesibilidad y diseño) y los propósitos de la interacción. Además no debe olvidarse que el principal factor de popularidad de una plataforma es su contenido, y en el caso de las redes sociales, éste viene determinado por la propia estructura de la red que permite crear contenidos junto a otros usuarios. Ambos tipos de red (MMORPG y Red Social ) se encuentran orientadas hacia un contenido de ocio "recreativo", puesto que se basan en el placer de crear, modificar y compartir contenidos entre los miembros de una red, ya sea en forma de un relato 
con tintes fantásticos (World of Warcraft) o la interacción social mediada entre amigos y conocidos (Facebook).

\section{Perfiles \\ 4.1.2 La creación de un nuevo cuerpo: Avatares, Personajes y}

El sujeto ha escogido el nido según sus intereses, motivado principalmente por el atractivo de sus contenidos. Pero, tarde o temprano, desea verse en él y formar parte de la comunidad. La integración en este nuevo entorno exige la creación de un nuevo cuerpo, proceso que entendemos como Creación de Avatares. En un principio, en los primeros años de una Internet poblada por comunidades on-line, (chats, MUDs, etc), la adopción de una identidad virtual era un acto de comunicación inconsciente (Turkle, 1995; 1997). El usuario adoptaba una máscara, foto o avatar, proyectando características propias de su personalidad y en muchas ocasiones de su físico. Más tarde, las comunidades de juego on-line se beneficiaron de un sistema gráfico más complejo que permitía la representación gráfica en tiempo real, con lo que las MUDs pasaron a ser MMORPG. En el ámbito del MMORPG, la creación de un avatar implica la creación de un personaje. Esta "personalidad tras la máscara" presupone una intención lúdica o fantasía de divertimento (Caillois, 1958). El usuario ha pasado a ser un jugador, y en esta suspensión pactada de la realidad que implica el juego, crea el personaje.

La creación de este personaje incluye la representación del mismo a través del avatar, así como una personalidad ficticia y un pasado propio que puede compararse a los detalles biográficos de una novela o película. Esta descripción de eventos "vividos" por el personaje y el jugador, refleja los aspectos narrativos que tiene el JdR como género lúdico. Algunos de ellos son producto de la imaginación del usuario, en un acto de enunciación similar a la narración de ficción. Otros en cambio, emergen de la secuencia de episodios que conforman la experiencia de juego o gameplay (Tronstad, 2001). Además, el avatar del MMORPG, como actualización del JdR y el VJdR define un aspecto esencial del género lúdico que relacionamos con la Mimicry de R. Caillois (1958): la configuración y actualización del personaje. De esta manera, la apariencia y sus habilidades serán alterados a través de la interacción con el entorno, adquiriendo herramientas, vestuario y armas de la misma forma que mejoran sus habilidades curativas o de lucha. La actualización de este avatar se asemeja al "traje inteligente" con el que se definió en un principio la Realidad Virtual (Laurel et al., 1994), puesto que se encuentra programado para ser una extensión del cuerpo del usuario y funciona como una interfaz adaptable al entorno.

Por su parte el Perfil (Página personal o profile) de una Red Social Virtual supone una forma de comunicación que comienza como una experiencia de emulación de las relaciones sociales y termina convirtiéndose en una nueva vía de socialización. Un buen ejemplo de esta evolución de las Redes Sociales lo tendríamos en la nueva versión de Facebook, tras su actualización en Diciembre de 2010. Lo que en principio definió su creador como "un modo de estar en contacto con tus amigos" ha sido transformado en una gran tarjeta de presentación ante otros usuarios. Mucho más visual y atractivo, su interfaz abandona las pestañas y reorganiza los contenidos. En 
éstos, las fotos ocupan un lugar primordial, ayudadas por breves apuntes que simulan una informal bionota y la información más importante: la red social personal. Ésta se encuentra presentada en forma de nodos ("Universidad", "montar en bici", "Lucía Martínez"...) los cuales conectan a usuarios y visitantes.

Sin embargo, la creación de un Perfil en redes sociales no puede compararse al diseño de personajes en otros marcos lúdicos como son los juegos de rol o MMORPGs. Si bien el perfil personal cuenta con los componentes principales del JdR (la configuración/actualización de los personajes y la narración colaborativa), carece del componente de competición (Agôn) y de un marco reglado (Ludus) que organice el juego, por lo que no puede considerarse como tal. Por otra parte, su expresión se encuentra más relacionada con la exploración de un espacio lúdico (similar al de un juego en comunidad) que al de otros géneros informativos o publicitarios. Ésta es, utilizando la terminología de Ryan (2004) una experiencia placentera, inmersiva, interactiva y basada en el diseño.

Como forma narrativa, en cambio, el perfil social presenta una forma equivalente a la de un relato de ficción, en el que los usuarios acometen el papel de enunciadores o narradores. En esta narración se recurre con frecuencia a otros códigos o lenguajes, tales como fotografías y videos de los usuarios, así como material referenciado de otras webs. Por si la intención de los autores de Facebook de construir una experiencia de ocio basada en el intercambio de relatos no hubiese quedado bastante clara a lo largo de la historia de la plataforma, en diciembre de 2011 se introdujo la Biografía (Timeline). Esta nueva aplicación permite la visualización del perfil personal en forma de una línea narrativa totalmente configurable. Así, el usuario puede contar "su propia historia" en un hipermedia que enlaza a otros nodos ("nacido en...") y perfiles ("casado con..."). La Biografía no sustituye al perfil habitual de Facebook, sino que supone una forma diferente de acceder a la misma información, ordenada de forma cronológica. La narración colectiva convive así con la construcción del personaje-perfil, tal y como sucedía en el Juego de Rol.

\section{CONCLUSIONES}

Finalmente, se revisan las ideas más importantes sobre la evolución del Juego de Rol (JdR) como género lúdico así como la influencia de las nuevas formas de literatura aparecidas a como consecuencia de la implantación del hipertexto y que hemos denominado "Narrativa Liquida":

- En el terreno de la comunicación actual, el usuario ha adquirido un papel central, del que enfatizamos las funciones ergódicas e interactivas (Landow, 1993; MartínBarbero, 2003; Ryan, 2006). Las consecuencias de esta evolución pueden verse en las formas actuales de Narrativa que llamaremos "Líquida" para emular la terminología de Bauman (2005; 2006). Esta "Narrativa Líquida" se encuentra representada en aspectos como la aleatoriedad, la inmersión y la reticularidad.

- Por otra parte, los marcos lúdicos entre los que destaca por su complejidad y la incursión de aspectos narrativos el Juego de Rol también han sufrido esa evolución 
gracias a la tecnología. Este hecho ha posibilitado la creación de entornos de inmersión directa para el Juego de Rol (Videojuegos de Rol) y nuevos géneros que enfatizan valores de creación y sentido de comunidad (MMORPGs).

- El Juego de Rol (JdR) y sus transformaciones digitales (VJdR y MMORPGs), siguen cumpliendo con las características principales de los juegos, pese al importante peso que tiene en ellos los aspectos narrativos (Juul, 2001; Klevjer, 2002; Frasca 2003). Así, siguiendo la teoría de análisis de los juegos de Roger Caillois (1958) todos presentan aspectos comunes (mimicry alea, âgon, ilinix,...) que los definen como entornos lúdicos y los colocan entre los dos extremos del juego: el marco reglado (ludus) y la fantasía de divertimento (paidea).

- Entre los numerosos entornos lúdicos que ha originado la ya comentada "revolución digital", las redes sociales virtuales están adquiriendo una notable presencia, debido a su popularidad y difusión. En ellas podemos observar características de esa Narrativa Líquida pero también y sobre todo, aspectos esenciales de la teoría de Caillois. Entre estos componentes destaca sobre todo el Mimicry (mimetismo), localizable en la customización que el usuario realiza de los perfiles en Redes Sociales Virtuales (Facebook). Por otra parte, este rasgo era ya patente en otras plataformas de orientación lúdica como los MMORPGs (World of Warcraft). Las redes sociales deberían ser entendidas no sólo como formas de comunicación y socialización, sino como formas evolucionadas de narración colectiva, en las cuales el usuario proyecta un relato propio de carácter autobiográfico.

En un futuro, se espera que estas reflexionen ayuden a perfilar una nueva definición del Juego de Rol como género híbrido dentro de los marcos lúdico y narrativo. Para este propósito, ayudaría la elaboración de una tipología actualizada del Juego de Rol y de todas sus derivaciones que dé soporte a esfuerzos anteriores (Dormans, 2006; Aarseth, 2007) y a la vez, oriente las investigaciones de otros productos lúdicos. Confiamos en que el usuario y su papel a través de perfiles o avatares, seguirá siendo el objeto principal de revolución pues es a través de lo lúdico, en el juego y la narrativa, donde el hombre puede redefinirse.

\section{REFERENCIAS}

Aarseth, E. (1997): Cybertext. Perspectives on Ergodic Literature. The Johns Hopkins University. Baltimore.

Aarseth, E. (2007). "Investigación sobre juegos: aproximaciones metodológicas al análisis de juegos" en Jugabilidad: arte, videojuegos y cultura. Artnodes. no 7. UOC. Disponible en http://www.uoc.edu/artnodes/7/dt/esp/aarseth.pdf (Consultado el: 12/01/2010).

Bauman, Z. (2006): Vida líquida. Editorial Paidós. Barcelona.

Bauman, Z. (2005): Amor líquido. Acerca de la fragilidad de los vínculos humanos. Ed. Fondo de Cultura Económica. Buenos Aires. 
Berenguer, X. (2007): "Historias por ordenador". Disponible en:

http://www.upf.edu/pdi/dcom/xavierberenguer/textos/histor/narrc.html (Consultado el: $12 / 02 / 2011)$

Breton, P. (2000): La utopía de la comunicación. Buenos Aires: Nueva Visión.

Caillois, R. (1958): Teoría de los Juegos. Seix Barral. Barcelona.

Carr, D.; Buckingham, D.; Burn, A. y Schott, G. (2006): Computer games: Text, narrative and play. Polity Press. London.

Chen, J. V. y Park, Y. (2005): "The Differences of Addiction Causes between Massive Multiplayer Online Game and Multi-User Domain" en International Review of Information Ethics. Vol. 4. Pág. 53-60.

Choi, D. y Kim, J. (2004): "Why people continue to play online games: In search of critical design factors to increase customer loyalty to online contents" en Cyber Psychology \& Behavior, no 7. Pág. 11-24.

Christakis, N. A. y Fowler, J. H. (2010): Conectados: el sorprendente poder de las redes sociales y cómo nos afectan. Taurus. Madrid.

Contreras, F. (1998): El cibermundo. La dialéctica del discurso informático. Alfar. Sevilla.

Dormans, J. (2006): "On the Role of the Die: A brief ludologic study of pen-and-paper roleplaying games and their rules" en Game Studies, vol. 6. Disponible en: http://gamestudies.org/0601/articles/dormans (Consultado el: 12/11/2010).

Entrevernes, Grupo de (1982): Análisis semiótico de los textos: Introducción, Teoría, Práctica. Ediciones Cristiandad. Madrid.

Eskelinen, M.: "The Gaming Situation" en Game Studies. Vol. 1 (1). Disponible en http://www.gamestudies.org (Consultado el: 12/11/2011).

Featherstone, M. y Burrows, R. (1995): "Cultures of Technological Embodiment: An Introduction", en Cyberspace/Cyberbodies/Cyberpunk. Cultures of Technological Embodiment (pp.1-19). Sage. London.

Fernback, J. (1999): "There Is a There There". En Jones, S.: Doing Internet Research. Critical Issues and Methods for Examining the Net (pp.203-301). Sage. Thousand Oaks, London \& New Delhi.

Frasca, G.: "Ludologists love stories, too: notes from a debate that never took place" en Digital Games Research Association (DiGRA, 2003). Disponible en http://www.gamesconference.org/2003/ (Consultado el: 18/12/2010). 
Garín, M. y Pérez, O. (2009): "Entre mundos e historias: ciencia ficción y experiencia de juego" en Formats: revista de comunicació audiovisual. n.5. Disponible en: http://www.upf.edu/materials/depeca/formats/art_dos6_esp.htm (Consultado el: 18/11/2011).

Gibson, W. (1986): Quemando Cromo. Minotauro. Barcelona.

Jorgensen, K. (2010): "Game Characters as Narrative Devices. A Comparative Analysis of Dragon Age: Origins and Mass Effect 2" en Eludamos. Journal for Computer Game Culture, n. 4 (2). Pág: 315-331.

Juul, J. (2001): "Games Telling stories? A brief note on games and narratives" en Game studies. N. 1. Pág: 1-12.

Koskimaa, R. (2000) Digital Literature. From Text to Hypertext and Beyond. Tesis doctoral de la Universidad de Jyväskylä. Disponible en http://www.cc.jyu.fi/ koskimaa/thesis (Consultado el: 18/12/2010).

Klevjer, R. (2002): "In Defence of Cutscenes". En Mäyrä, F.: Computer Games and Digital Cultures Conference Proceedings (pp. 191-202). Tampere University Press. Tampere.

Klevjer, R. (2007): What is the Avatar? Fiction and Embodiment in Avatar-Based Singleplayer Games. Phd thesis, University of Bergen. Disponible en http://folk.uib.no/smkrk/docs/RuneKlevjer_What\%20is\%20the\%20Avatar_finalprint.p df (Consultado el: 20/12/2010).

Landow, G. P. (1993): Hypertext. The Convergence of Contemporary Critical theory and Technology $\left(1^{\mathrm{a}}\right.$ ed.). The Johns Hopkins University Press. Baltimore.

Laurel, B.; Strickland, R.; Tow, R. (1994): "Placeholder: Landscape and Narrative in Virtual Environments" en Computer Graphics, vol. 28 (2). Pág: 118-126.

Lipovetsky, G. y Serroy, J. (2009): La pantalla Global: Cultura mediática y cine en la era hipermoderna. Anagrama. Barcelona.

Lotman, Y. (1996): La semiósfera. La semiótica de la cultura. Cátedra. Madrid.

Mabrouk, M.; Dykiel, R.; Henry J. y Pinon, J. M. (1991): "A hyperdocument model based on the ODA standard". Actas del congreso: Intelligent text and image handling, Barcelona.

Manninen, T. (2002): "Towards Communicative, Collaborative and Constructive Multiplayer Games". En Mäyrä, F. (ed.): Proceedings of Computer Games and Digital Cultures Conference ( pp. 155-169) Tampere University Press. 
Manovich, L. (2001): The Language of New Media. Cambridge, MIT Press. Massachusetts.

Martin, A. (2009): "Digital Literacy for the Third Age: Sustaining Identity in an Uncertain World" en elearning Papers, $\mathrm{n}^{\circ} .12$. Disponible en http://www.elearningeuropa.info/files/media/media18500.pdf (Consultado el: 120/11/2011).

Martín-Barbero, J. (2003): "Saberes hoy: diseminaciones, competencias y transversalidades" en Revista Iberoamericana de Educación. №. 32. Pág. 17-34.

Molina, D. (2009) "Final Fantasy XIII ofrecerá unas 50-60 horas de juego". 3D Juegos. Disponible en http://www.3djuegos.com/noticias-ver/108849/final-fantasy-xiiiofrecera-unas-5060-horas-de-juego/ (Consultado el: 16/12/2011).

Peinado, F. y Santorum, M. (2004): "Juego Emergente: ¿Nuevas formas de contar historias en videojuegos?". En Icono.14, no IV.

Disponible en http://www.icono14.net/revista/num4/index.html (Consultado el: 20/11/2011).

Ricaurte, P. y Ortega, E. (2008): "Facebook o los nuevos rostros de la socialidad", IX Congreso Latinoamericano de Investigación de la Comunicación. Disponible en http://www.alaic.net/alaic30/ponencias/cartas/Internet/ponencias/GT18_35Ricarte_Or tega.pdf (Consultado el: 20/10/2011).

Rodríguez, A. (2006): "¿Que es un (hiper)texto?" en Chartier, R. (2006): ¿Qué es un texto? Madrid: Círculo de Bellas Artes, pp. 87-100.

Ryan, M. L. (2004): La narración como realidad virtual, la inmersión y la interactividad en la literatura y en los medios electrónicos. Paidós Ibérica. Barcelona.

Susman, W. (1984): Culture as History: The Transformation of American Society in the Twentieth Century. Pantheon Books. New York.

Tosca, S. (2000): "The Player-Character in Computer Games". En DAC2000, Digital Arts and Culture Conference. Bergen, Norway, 2000.

Tosca, S. (2002): "The EverQuest Speech Community". En Mäyrä, F.: Proceedings of Computer Games and Digital Cultures Conference. Tampere: Tampere University Press, pp. 341-353.

Tosca, S. (2003): "The Quest Problem in Computer Games". En Technologies for Interactive Digital Storytelling and Entertainment (TIDSE). Darmstadt: Fraunhofer IRB Verlag. Disponible en http://www.itu.dk/people/tosca/quest.htm (Consultado el 23-122011)

Tronstad, R. (2001): "Semiotic and Non-Semiotic MUD Performance", COSIGN, 
Amsterdam.

Disponible en http://www.kinonet.com/conferences/cosign2001/pdfs/Tronstad.pdf (Consultado el: 18/12/2010).

Turkle, S. (1984): El segundo yo: las computadoras y el espíritu humano. Galápago. Buenos Aires.

Turkle, S. (1995): Life on the Screen: Identity in the Age of the Internet. Simon \& Schuster. New York.

Tychsen, A.; Heide-Smith, J.; Hitchens, M.; Tosca, S. (2006): "Communication in Multi-player Role Playing Games. The Effect of Medium". Technologies for Interactive Digital Storytelling and Entertainment Lecture Notes in Computer Science, 2006, Volumen 4326/2006, Pág. 277-288.

Vega, M. J. (2003): Literatura hipertextual y teoría literaria. Mare Nostrum. Madrid.

\section{Manuel Hernández Pérez}

Licenciado en Psicología (2004) y Publicidad y Relaciones Públicas por la Universidad de Murcia (2006). Tras la realización de un DEA en Neurociencias en esta misma institución, compatibiliza su actividad profesional en Comunicación Institucional con investigaciones en el área de la Publicidad y nuevos formatos audiovisuales. Actualmente realiza su trabajo de tesis, centrada en modelos de análisis para el estudio de la narrativa transmedial. 